\title{
A rate dependent experimental and numerical analysis of adhesive joints under different loading directions
}

\author{
Maria Lißner ${ }^{1}$, a, Enrique Alabort ${ }^{1}$, Borja Erice ${ }^{1}, \mathrm{Hao}_{\mathrm{Cui}}{ }^{2}$, and Nik Petrinic ${ }^{1}$ \\ ${ }^{1}$ Department of Engineering Science, University of Oxford, Parks Road, Oxford \\ OX1 3PJ, UK \\ ${ }^{2}$ School of Aerospace, Transport and Manufacturing, Cranfield University, Bedford \\ MK43 0AL, UK
}

Received 1 August 2017 / Received in final form 14 December 2018 Published online 10 September 2018

\begin{abstract}
Adhesively bonded joints subjected to dynamic loading demand reliable and accurate measurement techniques to capture their rate dependent traction-separation behaviour. Particularly challenging is the measurement of damage and failure processes which are fundamental to develop the corresponding cohesive zone models. This paper proposes a new experimental methodology for the characterisation of rate-dependent behaviour of adhesive joints. The results of the adopted approach have been used to calibrate a rate-dependent numerical cohesive model proposed to simulate the failure of adhesive joints. The procedure was benchmarked against quasi-static DCB and ENF experiments.
\end{abstract}

\section{Introduction}

The use of adhesive joints in industrial applications has been increasing significantly in recent years. Correspondingly, understanding the behaviour and performance of adhesive interfaces has become critical to allowing the development of new engineering designs of lightweight structures [1]. A variety of numerical methods have been developed to analyse fracture performance of bonded structures. Among them, the cohesive zone model (CZM) is widely used for the analysis of the adhesive joint failure [2-4]. The accuracy of these models relies heavily on the experimental characterisation of the behaviour of adhesives [5]. Despite considerable study at quasi-static loading rates [6,7], few results have been reported about the performance of adhesively bonded joints loaded dynamically. It is the aim of this paper to propose an approach that combines experimental characterisation and numerical modelling to predict the rate-dependent failure of adhesively bonded joints.

The cohesive zone model is defined by the relationship between the interfacial traction and the separation of bonded surfaces [8]. In order to determine the traction-separation law (TSL) the identification of the stiffness, the strength, the fracture toughness and their overall relationship are required $[9,10]$. Traditionally,

a e-mail: maria.lissner@eng.ox.ac.uk 
the characterisation of the TSL for adhesive joints has been carried out under quasistatic loading conditions. Standardised experiments of the double cantilever beam (DCB) [11], the end notched flexure (ENF) [12] and the mixed-mode bend (MMB) [13] are employed to measure the fracture toughness for the loading directions in mode I, mode II and mixed-mode respectively. However, those are often unsuitable for the characterisation of the dynamic behaviour of adhesive joints. For dynamic loadings, experiments based on the Izod and Charpy technique exist [14-16]. However, while these techniques can be useful for qualitative comparative studies, their results do not lead to reliable analysis of the fracture toughness due to their inability to isolate the inertia effects. In order to overcome this limitation, the Split Hopkinson Bar (SHB) apparatus [17] has been utilised as under dynamic equilibrium conditions, the measurements of specimen's boundary conditions are not affected by inertia effects. Nevertheless, for the characterisation of adhesively bonded interfaces using the SHB apparatus, optimal specimen designs that ensure uniform wave propagation are necessary. Yokoyama et al. $[18,19]$ developed a mode I specimen which fulfilled these requirements. Nevertheless, there is still a need for reliable designs for mode II and mixed-mode, but also a need to adapt the framework to the non-dynamic regimes.

With the above in mind, the rate-dependent characteristics of an adhesive joint have been measured, modelled, and benchmarked. First, new specimen designs for mode I, mode II and mixed-mode fracture were characterised under three different loading regimes: quasi static loading rate, medium loading rate and high loading rate. Second, numerical modelling was used to simulate and calibrate a cohesive zone model using the finite element package LS-DYNA. Third, experimental analysis was used to quantify the rate-dependency of the joint in terms of stress, displacement and dissipated energy. Finally, the calibrated model was benchmarked against standardised experimental data obtained using the double cantilever beam (DCB) for mode I and the end notch flexure (ENF) experiments for mode II deformation.

\section{Materials and experimental methods}

The investigated specimens consisted of two titanium alloy Ti-6Al-4V end caps which were adhesively joined with the thermosetting epoxy film adhesive AF 163-2OST. The adhesive is supported with a glass fibre matt and it was sourced from $3 \mathrm{M}$ Scotch-Weld ${ }^{\mathrm{TM}}$. The area of application for this specific adhesive is mainly high performance applications in the aerospace and automotive sectors. This adhesive can be used to bond both similar (e.g. metal-to-metal) and dissimilar (metal-to-CFRP) materials. The thickness of the adhesive measured $0.12 \mathrm{~mm}$. In order to obtain an optimal adhesive joint, the surfaces of the titanium end caps were sand-blasted, cleaned and anodised prior bonding, [20]. In order to control accurately the thickness of the bondline, a custom designed bonding fixture and shims were employed. Appropriate specimen geometries for the characterisation of the adhesive joint behaviour for each loading mode had to be designed specifically with high-rate loading in mind.

The specimens were designed so that an impedance match between the loading bars and the end caps could be achieved (Fig. 1). The mode I specimens were designed as a butt joint with $4 \mathrm{~mm}$ thick end caps - see Figure 1a. The mode II were designed as a single lap shear with an overlap length of $10 \mathrm{~mm}$ - see Figure 1b. For the mixedmode specimens, the end caps were manufactured with a $45^{\circ}$ angle surface - see Figure 1c.

\subsection{Experimental setups}

The rate-dependent characterisation of the present adhesive joints had been carried out using three different experimental setups in tension. High-rate (HR) was 


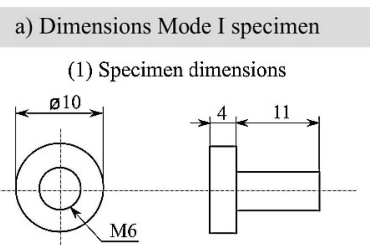

(2) Specimen manufactured

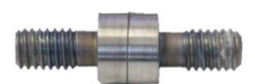

b) Dimensions Mode II specimen

(1) Specimen dimensions

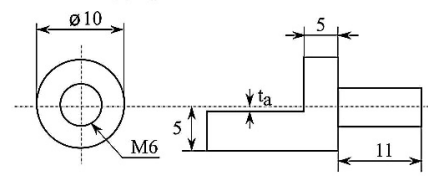

(2) Specimen manufactured

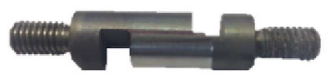

c) Dimensions Mixed-Mode specimen

(1) Specimen dimensions

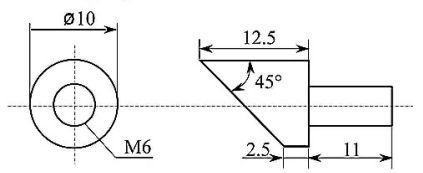

(2) Specimen manufactured

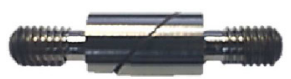

Fig. 1. Overview of the specimen geometries for different loading directions: (a) mode I loading direction, (b) mode II loading direction, and (c) mixed-mode loading direction.

a) Split Hopkinson Tensile Bar

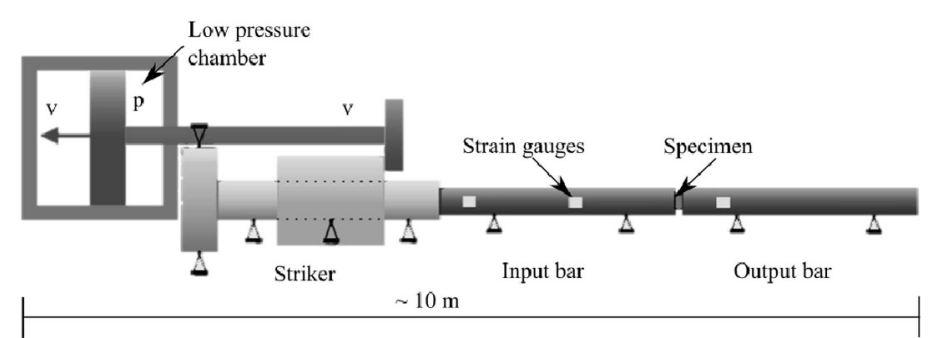

b) Quasi- static and Medium-rate test setup

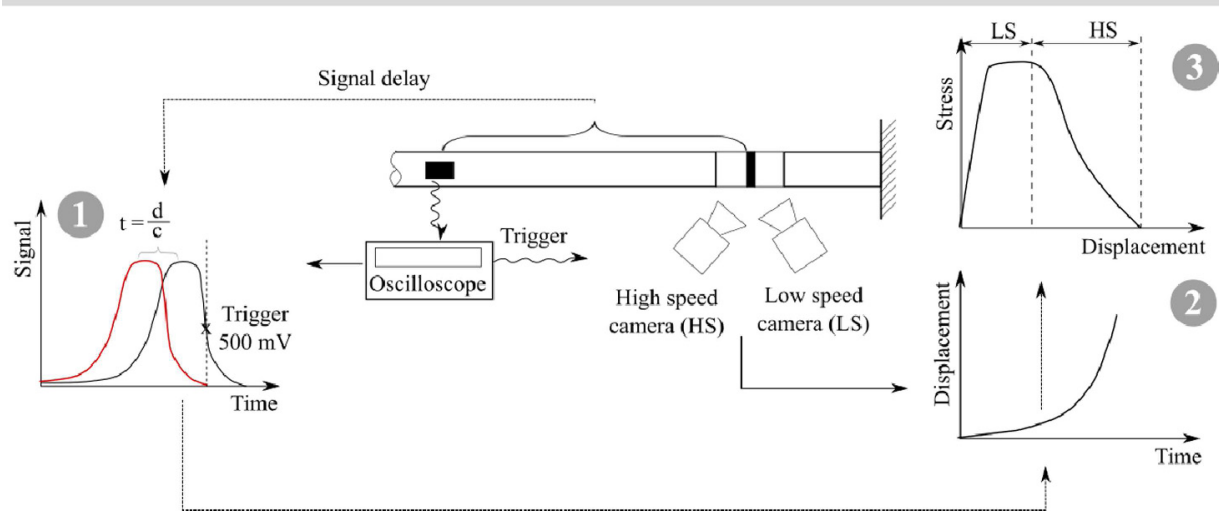

Fig. 2. Schematics of the experimental setups: (a) Split Hopkinson Tensile Bar [21] and (b) working principle of the modified setup for quasi-static and medium-rate experiments.

performed on a Split Hopkinson Tensile Bar (SHTB) apparatus [21]. In this case, the specimens were subjected to a loading velocity of $v=3000 \mathrm{~mm} / \mathrm{s}$. Figure 2 a shows the schematics of the SHTB: the loading device consists of a hollow striker which hits an anvil at the end of the input bar to produce the tensile load. On the other side of the specimen, the output bar is located. Strain gauges of the type FLA-2-8 from TML attached to the bars were used to measure the signal-time histories at an acquisition rate of 100 kilo-samples per second. An SI-Kirana high-speed photographic camera at the frame rate of $500000 \mathrm{fps}$ was employed to record $924 \times 768$ pixels resolution images for measurements of the separation of bonded surfaces by means of digital image correlation (DIC). 

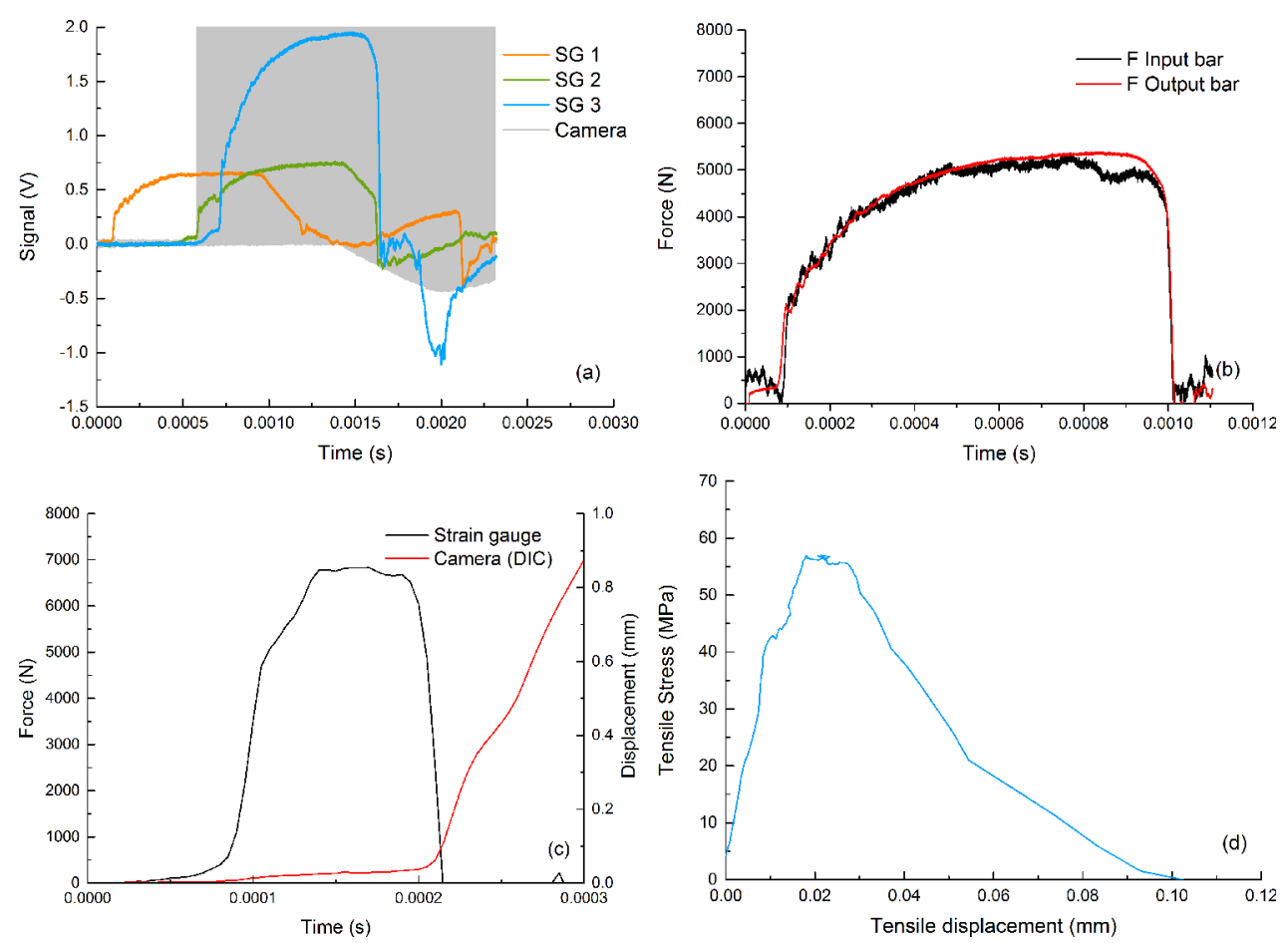

Fig. 3. (a) Signal history recorded by the oscilloscope during a high-strain rate test, (b) force equilibrium, (c) force and displacement synchronisation and (d) resultant stressdisplacement curve.

The quasi-static (QS) experiments were carried out on a Zwick screw-driven machine with a velocity of $v=0.05 \mathrm{~mm} / \mathrm{s}$. An Instron servo-hydraulic machine was employed for the medium-rate (MR) experiments. Here, the applied velocity was $v=10 \mathrm{~mm} / \mathrm{s}$. Figure $2 \mathrm{~b}$ shows the procedure developed to measure the softening curve on the MR and QS experiments. The setup follows a similar approach as in the high-rate (HR) experiments where a long bar with attached strain gauges was used to measure the softening slope of the stress-displacement curve. A camera system consisting of two cameras - a low-speed and a high-speed one - was used to measure the displacement of the loading and the fracture regimes respectively. The slow- and high-speed cameras recorded for the QS tests $706 \times 872$ pixels resolution images with $5 \mathrm{fps}$ and $924 \times 768$ pixels resolution images with $1000000 \mathrm{fps}$, respectively. For the MR experiments only the slow-speed camera has been changed to a SA 5 Photron to record $512 \times 408$ pixels resolution images with a frame rate of $65000 \mathrm{fps}$. The separation of titanium end caps during the tests was analysed using the images with digital image correlation method, and then synchronized with the loading signal.

In detail, the data of force and displacement of the loading part in the MR and QS tests can be synchronised straight forward since both mechanical test and image acquisition start simultaneously. In order to capture the falling slope of MR and QS tests, the signal of strain gauge attached to the output bar is used to trigger the high-speed camera and the lighting system. An analogous setup is used to capture the event during the HR characterisation. A typical strain gauge signal is shown in Figure 3a. This allows the synchronisation of the displacement data with the force values after including a delay in the signal. In particular, the stress wave - which starts from the sample - requires certain time to reach the strain gauge which is placed at 

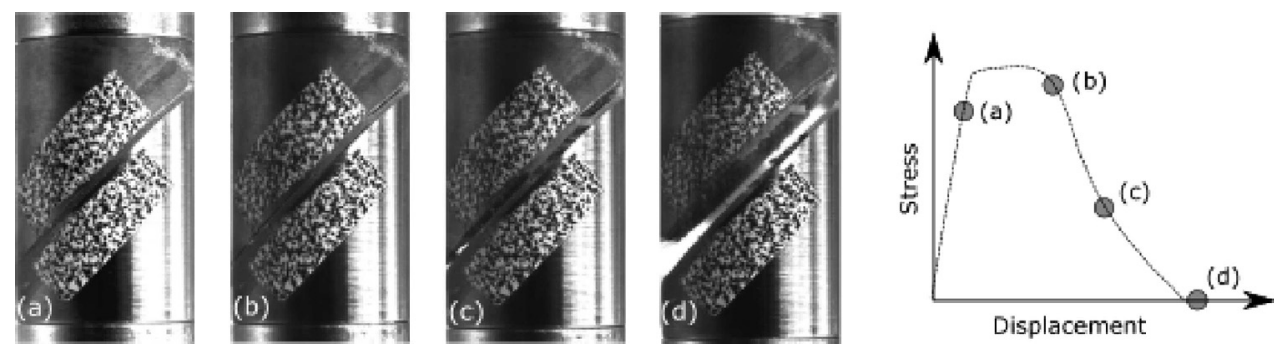

Fig. 4. Fracture process of a mixed-mode specimen captured using a high-speed camera showing the different stages: (a) elastic stage, (b) onset of failure, (c) crack propagation and (d) final failure.

an arbitrary distance from the sample. Figure 3c demonstrates the synchronised force and displacement data over time. After synchronisation, the final traction-separation curves can be generated - see Figure 3d.

\subsection{Results and discussion}

A series of tension tests at three different loading rates and at three different directions with respect to the adhesively bonded surfaces have been performed. An image sequence of a representative failure process of the experiments regardless of the loading direction is demonstrated in Figure 4. The experimental results are shown in Figure 5. The existence of a force equilibrium has been positively identified after each successful test - see Figure 3b. The initial elastic response was observed and consequently classed as relatively independent on the loading rate for mode I and mixed mode while for mode II, noticeable rate-dependency was observed.

The graphs show that the stress-separation curves have a trapezoidal shape, this is independent of the loading mode. However, the strength is clearly dependent on the loading rate since: it increases while increasing the velocity. Additionally, it is shown that the final failure separation decreased with increasing deformation rate. The calculated dissipated energy showed the tendency to decrease with increasing loading rates. As expected, the results of the mixed-mode adhesive joints lay right between mode I and mode II. A summary of the experimental characterisation is detailed in Table 1. Due to the occurrence of oscillation, the maximum strength of each experiment was obtained by averaging the value of the five highest data points. The statistical measurements have been derived from three repeated tests for each loading rate and loading direction, therefore, careful consideration is advised when rationalising the values of standard deviation.

The results represented in Table 1 for the three loading directions show that the strength was significantly higher under dynamic than under quasi-static conditions. However, the dissipated energy showed a slight increase between QS and MR, but a slight decrease for HR. Additionally, it is observed that the value of the strength for mixed-mode loading is close to the values of mode II loading whereas the dissipated energy is closer to that calculated for mode I.

\section{Numerical analysis}

Numerical simulations of the experimental investigation were performed using the explicit non-linear finite element code LS-DYNA. The finite element models for 

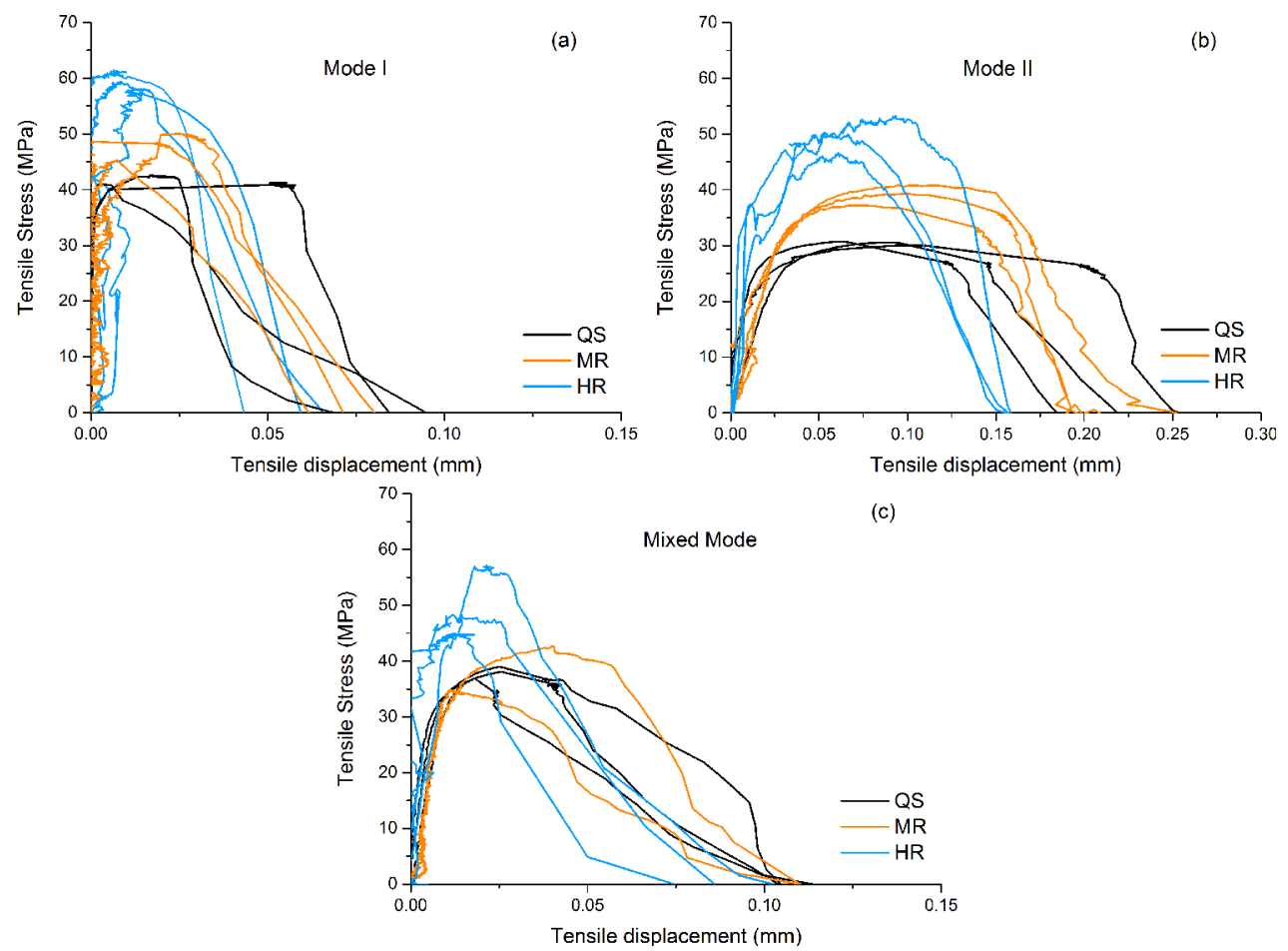

Fig. 5. Experimental tensile test results for the titanium-titanium adhesive joints with an adhesive thickness $t_{a}=0.1 \mathrm{~mm}$ under quasi-static, medium-rate and high-rate loading in mode I (a), mode II (b) and mixed-mode (c) loading direction.

Table 1. Mean properties( \pm standard deviation) for adhesive joints in mode I, II and mixedmode loading under quasi-static, medium-rate and high-rate loading.

\begin{tabular}{lllcl}
\hline & & $\begin{array}{l}\text { Strength } \\
{[\mathrm{MPa}]}\end{array}$ & $\begin{array}{c}\text { Final displ. } \\
{[\mathrm{mm}]}\end{array}$ & $\begin{array}{l}\text { Dissipated energy } \\
{[\mathrm{N} / \mathrm{mm}]}\end{array}$ \\
\hline Mode I & $0.05 \mathrm{~mm} / \mathrm{s}$ & $41 \pm 0.9$ & $0.08 \pm 0.01$ & $2.0 \pm 0.7$ \\
& $10.0 \mathrm{~mm} / \mathrm{s}$ & $48 \pm 2.7$ & $0.07 \pm 0.01$ & $2.1 \pm 0.6$ \\
& $3000 \mathrm{~mm} / \mathrm{s}$ & $59 \pm 1.5$ & $0.06 \pm 0.01$ & $1.7 \pm 0.8$ \\
Mode II & & & & \\
& $0.05 \mathrm{~mm} / \mathrm{s}$ & $30 \pm 0.3$ & $0.22 \pm 0.03$ & $5.0 \pm 1.1$ \\
& $10.0 \mathrm{~mm} / \mathrm{s}$ & $39 \pm 2.1$ & $0.21 \pm 0.03$ & $6.0 \pm 0.7$ \\
Mixed-mode & $0.05 \mathrm{~mm} / \mathrm{s}$ & $38 \pm 1.3$ & $0.11 \pm 0.01$ & $2.4 \pm 0.5$ \\
& $10.0 \mathrm{~mm} / \mathrm{s}$ & $40 \pm 4.3$ & $0.11 \pm 0.04$ & $2.5 \pm 0.6$ \\
& $3000 \mathrm{~mm} / \mathrm{s}$ & $49 \pm 6.1$ & $0.09 \pm 0.01$ & $2.2 \pm 0.6$ \\
\hline
\end{tabular}

mode I, mode II and mixed-mode experiments included only the specimen geometry and were modelled using 8-node single integration point hexahedral elements with stiffness-based hourglass control - see Figure 6a. The end caps were assumed linear elastic using mechanical properties of the titanium alloy Ti-6Al-4V: $E=114 \mathrm{GPa}$, $\varrho=4.43 \times 10^{-6} \mathrm{~kg} / \mathrm{mm}^{3}$ and $\nu=0.34$. Since this titanium alloy has a yield strength of approximately $800 \mathrm{MPa}$, almost 15 times higher than the maximum achievable adhesive strength $(\sigma=60 \mathrm{MPa})$, one would assume that the response of the metal 
a) Model description

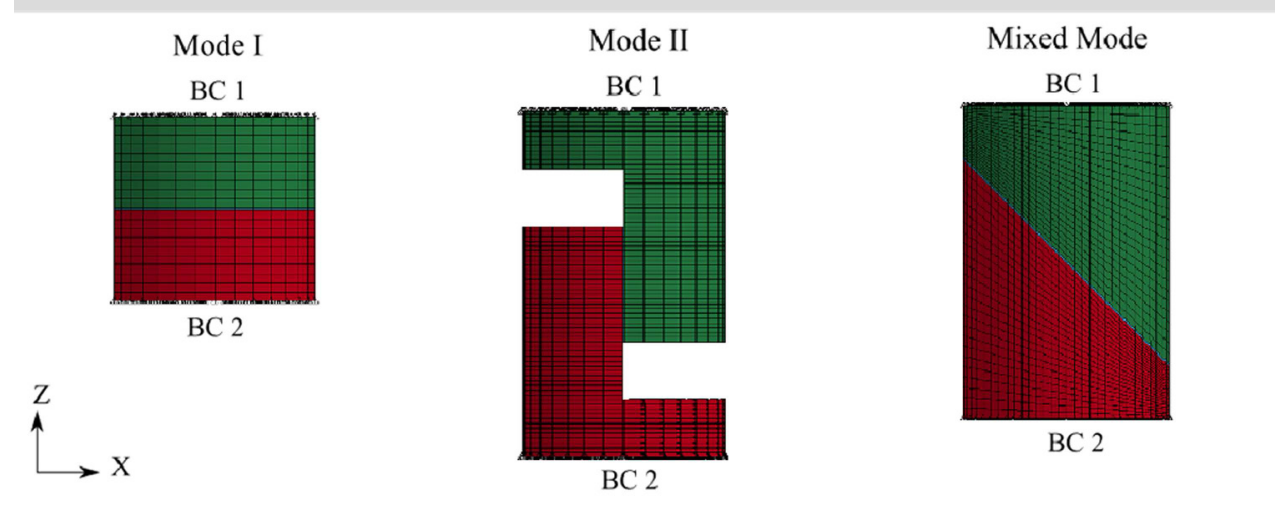

b) High-rate boundary conditions
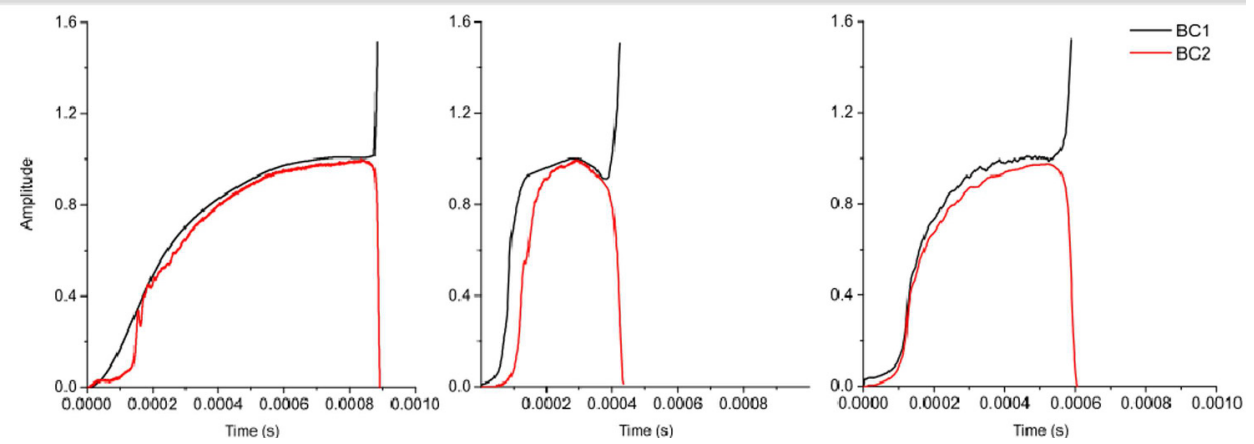

Fig. 6. Finite element model details: (a) model description for each loading direction and (b) boundary conditions for the high-rate loading case.

end caps is purely elastic. The adhesive layer was modelled using cohesive elements in combination with an already existent rate dependent tri-linear traction separation law (TSL) implemented in LS-DYNA.

The boundary conditions were extracted directly from the real experimental data obtained from representative curves for each loading regime and loading direction. These were applied as boundary conditions (BC) in the numerical models to simulate the different loading regimes. In particular, for the HR experiments, the velocity histories measured using digital image correlation from the input and output bars during the experiments were applied to the end cap faces as shown in Figure $6 \mathrm{~b}$. For the QS and MR experiments, a smooth ramp velocity history was applied on one end cap face nodes - this was believed to mimic the constant displacement applied on those experiments. The second end cap nodes were fixed in the translationally. For the latter simulations, mass scaling was employed so that the stable time increment $\Delta t=t_{f} / 10^{5}$ was calculated with $t_{f}=12 \mathrm{~s}$ being the time corresponding to the total calculation time.

\subsection{Rate dependent cohesive zone model}

The material model "MAT 240" in LS-DYNA [22] allows the modelling of a mixed-mode, rate dependent, elastic ideally plastic tri-linear TSL. The stress rate 
dependency for mode I and II strengths follows a linear logarithmic function written as

$$
t_{\mathrm{n}}\left(\dot{\varepsilon}_{\mathrm{eq}}\right)=\left|t_{\mathrm{n}, 0}\right|+\left|t_{\mathrm{n}, 1}\right| \max \left(0, \ln \frac{\dot{\varepsilon}_{\mathrm{eq}}}{\dot{\varepsilon}_{\mathrm{n}}}\right)
$$

and

$$
t_{\mathrm{t}}\left(\dot{\varepsilon}_{\mathrm{eq}}\right)=\left|t_{\mathrm{t}, 0}\right|+\left|t_{\mathrm{t}, 1}\right| \max \left(0, \ln \frac{\dot{\varepsilon}_{\mathrm{eq}}}{\dot{\varepsilon}_{\mathrm{t}}}\right)
$$

where $t_{\mathrm{i}, 0}$ are the QS strength reference values, $t_{\mathrm{i}, 1}$ describe the rate dependent values, $\dot{\varepsilon}_{\text {eq }}$ is the updated equivalent strain rate and $\dot{\varepsilon}_{\mathrm{i}}$ are the reference strain rates for $i=n, t$ which represent mode I and mode II respectively. Analogously, the energy rate dependency is described using an exponential function following

$$
\left.G_{\mathrm{I}}^{\mathrm{C}}\left(\dot{\varepsilon}_{\mathrm{eq}}\right)=\left|G_{1,0}\right|+G_{1, \infty}-\left|G_{1,0}\right|\right) \exp \left(-\frac{\dot{\varepsilon}_{\mathrm{G}_{1}}}{\dot{\varepsilon}_{\mathrm{eq}}}\right),
$$

and

$$
\left.G_{\mathrm{II}}^{\mathrm{C}}\left(\dot{\varepsilon}_{\mathrm{eq}}\right)=\left|G_{2,0}\right|+G_{2, \infty}-\left|G_{2,0}\right|\right) \exp \left(-\frac{\dot{\varepsilon}_{\mathrm{G}_{2}}}{\dot{\varepsilon}_{\mathrm{eq}}}\right),
$$

where equations (3) and (4) represent mode I and mode II, respectively.

The mixed-mode behaviour is defined by a quadratic criteria for the yielding and the damage initiation. The mixed-mode yield initiation displacement $\delta_{\mathrm{m} 1}$ and damage initiation displacement $\delta_{\mathrm{m} 2}$ were calculated using the expression

$$
\delta_{\mathrm{mi}}=\delta_{\mathrm{ni}} \delta_{\mathrm{ti}} \sqrt{\frac{1+\beta^{2}}{\delta_{\mathrm{ti}}^{2}+\left(\beta \delta_{\mathrm{ni}}\right)^{2}}}, \quad i=1,2,
$$

where $\beta=\frac{\delta_{\mathrm{ti}}}{\delta_{\mathrm{ni}}}$ represents the mixed-mode ratio whereas $i=1,2$ represent the yield initiation and the damage initiation displacement respectively. These parameters are described following

$$
\delta_{\mathrm{n} 1}=\frac{t_{\mathrm{n}}\left(\dot{\varepsilon}_{\mathrm{eq}}\right)}{E} \quad \text { and } \quad \delta_{\mathrm{t} 1}=\frac{t_{\mathrm{t}}\left(\dot{\varepsilon}_{\mathrm{eq}}\right)}{G}
$$

and

$$
\delta_{\mathrm{n} 2}=\delta_{\mathrm{n} 1}+\frac{F_{\mathrm{G} 1} G_{\mathrm{I}}^{\mathrm{C}}\left(\dot{\varepsilon}_{\mathrm{eq}}\right)}{t_{\mathrm{n}}\left(\dot{\varepsilon}_{\mathrm{eq}}\right)} \quad \text { and } \quad \delta_{\mathrm{t} 2}=\delta_{\mathrm{t} 1}+\frac{F_{\mathrm{G} 2} G_{\mathrm{II}}^{\mathrm{C}}\left(\dot{\varepsilon}_{\mathrm{eq}}\right)}{t_{\mathrm{t}}\left(\dot{\varepsilon}_{\mathrm{eq}}\right)}
$$

The parameters $F_{\mathrm{G} 1}=\frac{G_{p l}}{G_{I C}}$ and $F_{\mathrm{G} 2}=\frac{G_{p l}}{G_{I I C}}$ represent the proportion of the plastic energy over the whole dissipated energy which is defined as a constant which changes with the loading rate.

The final failure displacement is then calculated using the angle of the mixedmodity $\gamma$ which using a power law which can be written as

$$
\delta_{\mathrm{mf}}=\frac{\delta_{\mathrm{m} 1}\left(\delta_{\mathrm{m} 1}-\delta_{\mathrm{m} 2}\right) E G_{\mathrm{II}}^{\mathrm{C}} \cos ^{2} \gamma+G_{\mathrm{I}}^{\mathrm{C}}\left(2 G_{\mathrm{II}}^{\mathrm{C}}+\delta_{\mathrm{m} 1}\left(\delta_{\mathrm{m} 1}-\delta_{\mathrm{m} 2}\right) G \sin ^{2} \gamma\right)}{\delta_{\mathrm{m} 1}\left(E G_{\mathrm{II}}^{\mathrm{C}} \cos ^{2} \gamma+G G_{\mathrm{I}}^{\mathrm{C}} \sin ^{2} \gamma\right)} .
$$


Table 2. Identified parameters for the rate-dependent traction-separation law implemented in Ls-Dyna.

\begin{tabular}{ccccccc}
\hline $\begin{array}{c}\text { Density }\left(t / \mathrm{mm}^{3}\right) \\
1.2 \mathrm{e}-9\end{array}$ & $E(\mathrm{MPa})$ & $G(\mathrm{MPa})$ & $t_{a}(\mathrm{~mm})$ & & & \\
\hline$G_{1,0}$ & 1100 & 410 & 0.1 & & & \\
-3.00 & $G_{1, \infty}$ & $\dot{\varepsilon}_{\mathrm{G}_{1}}$ & $t_{\mathrm{n}, 0}$ & $t_{\mathrm{n}, 1}$ & $\dot{\varepsilon}_{\mathrm{n}}$ & $F_{\mathrm{G} 1}$ \\
& 2.00 & 0.10 & -36.00 & -1.80 & 0.10 & 0.50 \\
\hline$G_{2,0}$ & $G_{2, \infty}$ & $\dot{\varepsilon}_{\mathrm{G}_{2}}$ & $t_{\mathrm{t}, 0}$ & $t_{\mathrm{t}, 1}$ & $\dot{\varepsilon}_{\mathrm{t}}$ & $F_{\mathrm{G} 2}$ \\
-9.00 & 6.50 & 0.10 & -32.00 & -1.64 & 0.10 & 0.80 \\
\hline
\end{tabular}

The material parameters of the traction separation law were identified using an inverse modelling which employs the optimisation software package LS-OPT [23]. The calibrated material constants for the used cohesive zone model are shown in Table 2.

\subsection{Modelling results}

The numerically obtained traction-separation curves are shown as thick solid lines over the shaded area that represents the experimental results - see Figure 7 . The processed numerical results have been determined in an analogous manner to the experiments - the nominal traction is calculated by extracting the reaction force from a reference point assigned to a node of the solid end cap. The simulation of the characterisation tests with the calibrated material card "MAT 240" are in reasonable agreement with the experimental results and prove the usefulness of our characterisation framework. Moreover, the results of experiments with different loading directions allow one to define a failure envelope. This envelope can then be used to predict the failure of an adhesive joint at different loading rates and arbitrary interface orientations with respect to the loading axis. For the present case, Figure 8 shows the failure envelopes obtained experimentally as scatter dots and the numerical interpretation as dashed lines. These are represented as a function of the strengths $t_{\mathrm{n}}$ and $t_{\mathrm{t}}$, and the dissipated energies $G_{\mathrm{n}}$ and $G_{\mathrm{t}}$ - where the subindices $n$ and $t$ indicate the loading directions for mode I and mode II respectively. In general, the model shows a slight over-prediction of failure of both strength and dissipated energy for the different loading rates but these are well within the expected uncertainty.

\section{Benchmarking}

In order to demonstrate the potential and the accuracy of the proposed framework for measuring directly the traction-separation curve of an adhesive joint, two test cases have been modelled and compared to standardised experiments from the literature [24]: (i) the double cantilever beam (DCB) test and (ii) the end notched flexure (ENF) test. The former is used to calculate the fracture toughness in mode I direction and the latter to calculate it in mode II direction. Both were carried out using the adhesive AF 163-2K. The main difference of the AF 163-2OST and the AF 163-2K is the design of support carrier that is used for improving handling of larger scale applications. Reportedly, the design of the support has a minimum effect on the response of the adhesive itself. However, experimental data is available exclusively under QS conditions, thus allowing for this benchmarking to be conducted at the lowest loading rate only. 


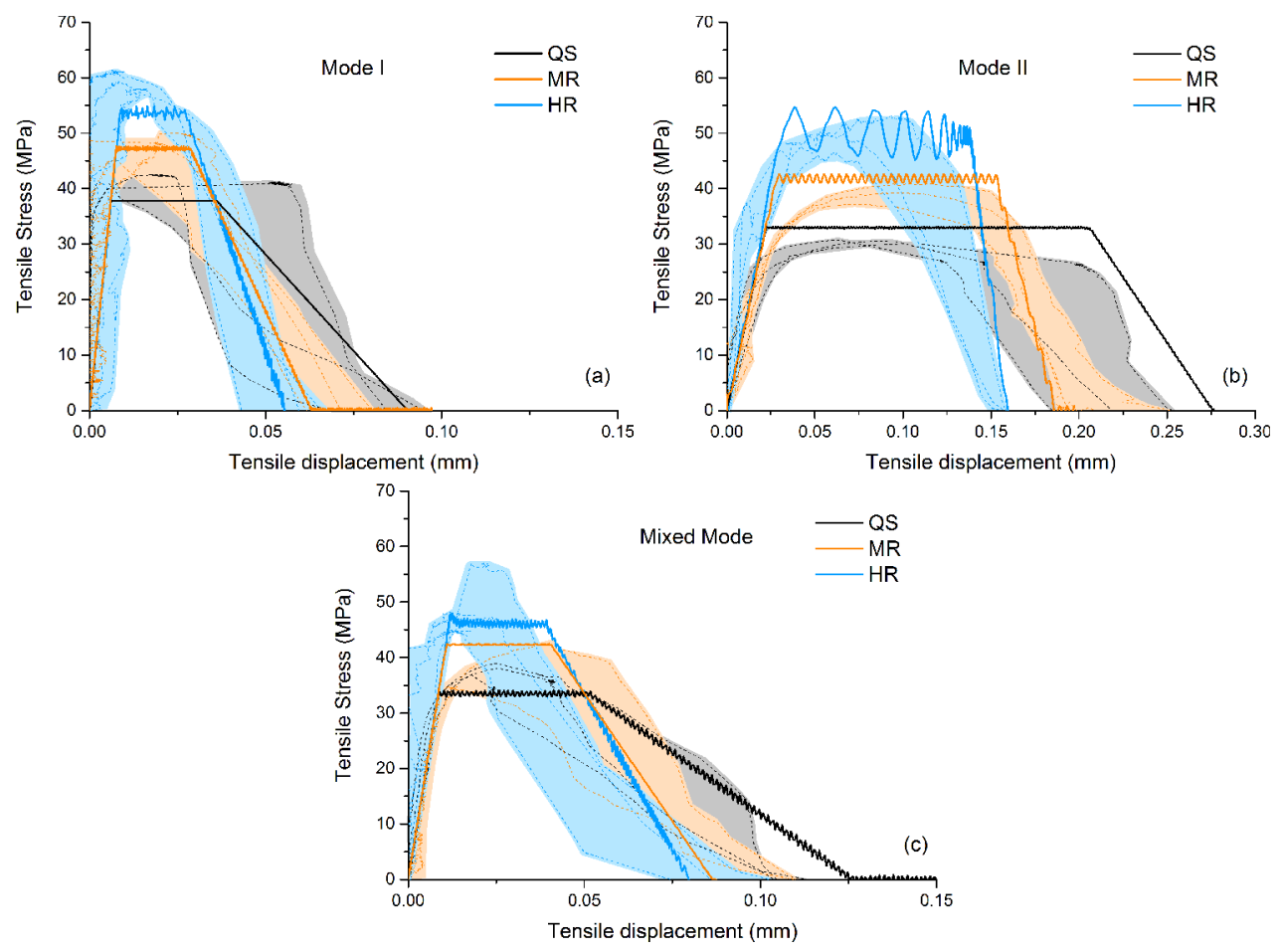

Fig. 7. Numerical tensile test results for the titanium-titanium adhesive joints with an adhesive thickness $t_{a}=0.1 \mathrm{~mm}$ under quasi-static, medium-rate and high-rate loading in mode I (a), mode II (b) and mixed-mode (c) loading direction.
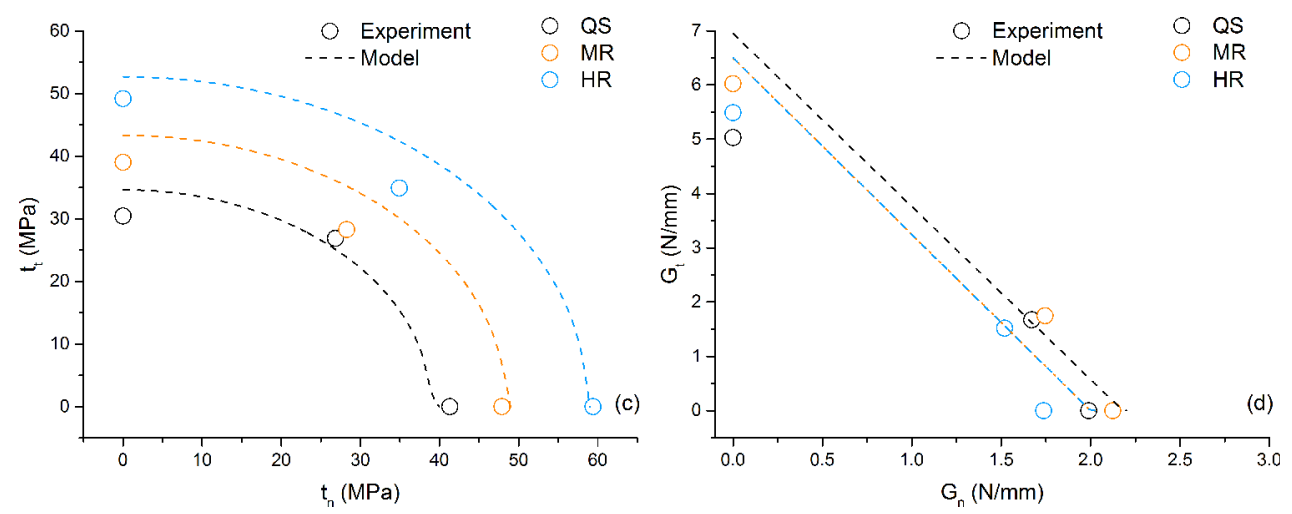

Fig. 8. Failure envelope for the stress (a) and dissipated energy (b).

\subsection{DCB with calibrated material model}

The geometry of the finite element model of the DCB test follows the dimension found in [24]. The adherents were modelled analogously to Section 3, with the properties of a typical aluminium alloy 2024-T3 Alclad $E=66 \mathrm{GPa}, \sigma_{y}=350 \mathrm{MPa}$ and $\nu=0.33$ [24]. The adhesive layer with a bondline thickness of $t_{a}=0.2 \mathrm{~mm}$ was modelled using the cohesive zone model introduced in Section 3. A pre-crack of $15 \mathrm{~mm}$ was introduced as described in [24]. The DCB was then pulled at a constant velocity of $v=0.2 \mathrm{~mm} / \mathrm{min}$. 

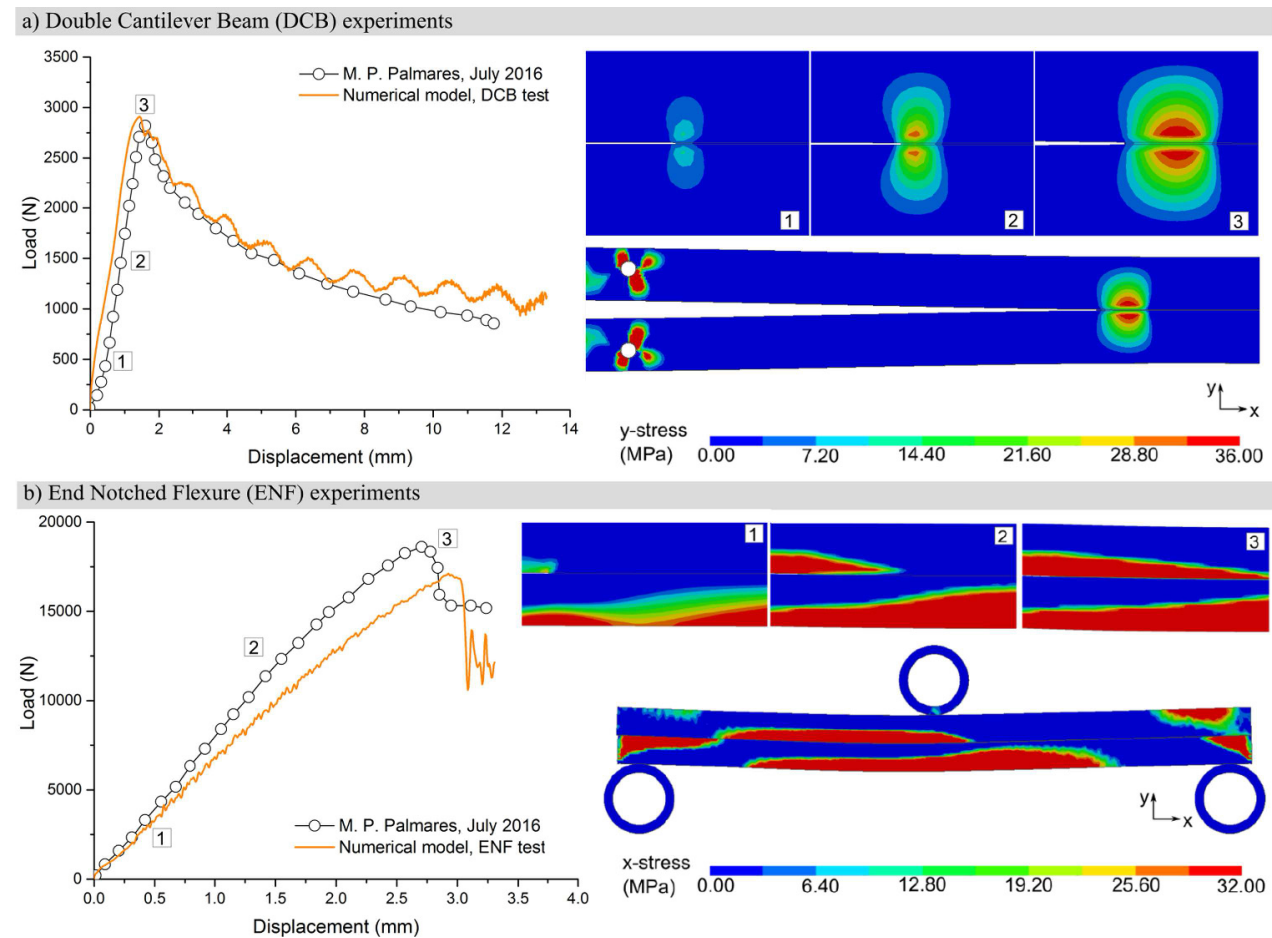

Fig. 9. Application of (a) DCB and (b) ENF experiments.

The results are shown in Figure 9. The finite element model with the calibrated material card is in excellent agreement with the experiments. These results clearly show that the proposed methodology is indeed valid for the mode I characterisation of adhesive joints under the investigated loading rate.

\subsection{ENF with calibrated material model}

Similar to Section 4.1, the numerical model for the ENF validation tests has been created. Analogously, the same cohesive law has been employed to model the adhesive layer. As described in [24], a pre-crack for the ENF tests of $50 \mathrm{~mm}$ was applied. The three-point bending experiment was simulated using a constant velocity of $v=0.2 \mathrm{~mm} / \mathrm{min}$. The obtained results show a difference in the stiffness about $15 \%$. This may be due to the difference in material properties between the adherents or the adhesive modelled here and those measured in the literature. Additionally, it may be argued that the displacement in the literature was measured with the cross-head of the machine, while here in the simulation the displacement has been measured from the loading pin. Nevertheless, similarly to the DCB, the presented ENF benchmarking justifies the use of the proposed methodology for the mode II characterisation of adhesive joints loaded at QS loading rates.

\section{Conclusions}

The rate dependent behaviour of an adhesive joint has been studied under three different loading directions - mode I, mode II and mixed-mode - using a newly proposed characterisation methodology. Experimental results were used to calibrate 
a cohesive zone model which was then benchmarked against results of standardised experimental methodologies. The following specific conclusions can be drawn:

1. The experimental characterisation of adhesive joints has been carried out using a new methodology. The modified experimental setups - using long bars - in the low rate regimes (QS and MR) enables the use of the stress wave propagation, similar to the SHTB, to measure the forces sustained by the adhesively bonded joints while isolating any inertia effects. In this regard, the same specimen geometry for all investigated loading regimes offers the opportunity to compare directly the rate dependent results. Additionally, the camera setups two cameras for the low rate regimes and one camera for high-speed loading - were critical for the measurement of the displacement in order to generate a complete traction-separation curve and the resulting whole dissipated energy.

2. The characterised adhesive - a thermosetting epoxy film adhesive AF 163-2OST - shows important rate dependency on the maximum values of strength an dissipated energy. More importantly, these values are also dependent on the mode of fracture.

3. These rate and mode dependent results were successfully represented using a tri-linear traction separation law using an implemented cohesive zone model in LS-DYNA. Mode I and mode II experimental results were used for parameter calibration while mixed-mode results were used as a benchmarking exercise.

4. For benchmarking, results from standardised quasi-static fracture experiments were used. Numerical simulations of the DCB and ENF tests show that the proposed experimental framework and subsequent cohesive zone model accurately captures the behaviour of the adhesive under more complex loading conditions.

The authors of this paper are grateful to Dr. Bamber Blackman and Dr. Rodolfo Rito, Imperial College London, for preparing the film-adhesive joints. Furthermore, the authors are grateful to Jeffrey Fullerton and Stuart Carter, Impact Engineering Laboratory, University of Oxford, for their assistance in the manufacturing of the specimens. The authors acknowledge the funding from Rolls-Royce plc which enabled this investigation.

Open Access This is an open access article distributed under the terms of the Creative Commons Attribution License (http://creativecommons.org/licenses/by/4.0), which permits unrestricted use, distribution, and reproduction in any medium, provided the original work is properly cited.

\section{References}

1. A. Kinloch, M. Little, J. Watts, Acta Mater. 48, 4543 (2000)

2. A. Needleman, J. Appl. Mech. 54, 525 (1987)

3. T. Ungsuwarungsri, W.G. Knauss, Int. J. Fract. 35, 221 (1987)

4. V. Tvergaard, J. Hutchinson, J. Mech. Phys. Solids 40, 1377 (1992)

5. H. Cui, Composites B 92, 193 (2016)

6. B.F. Sørensen, Acta Mater. 50, 1053 (2002)

7. H. Cui, S. Koussios, Y. Li, A. Beukers, Eng. Fract. Mech. 127, 235 (2014)

8. G. Geißler, M. Kaliske, Eng. Fract. Mech. 77, 153 (2010)

9. M. May, O. Hesebeck, Eng. Fail. Anal. 56, 441 (2015)

10. G. Alfano, Compos. Sci. Technol. 66, 723 (2006)

11. I.A. Ashcroft, D.J. Hughes, S.J. Shaw, Int. J. Adhes. Adhes. 21, 87 (2001) 
12. Q.D. Yang, M.D. Thouless, S.M. Ward, Int. J. Solids Struct. 38, 3251 (2001)

13. J.R. Reeder, J.R. Crews Jr., AIAA J. 28, 1270 (1990)

14. F. Kadioglu, R.D. Adams, Int. J. Adhes. Adhes. 56, 73 (2015)

15. J. Casas-Rodriguez, I. Ashcroft, V. Silberschmidt, J. Sound Vibrat. 308, 467 (2007)

16. W. Hufenbach, F. Marques Ibrahim, A. Langkamp, R. Bøhm, A. Hornig, J. Sound Vibrat. 308, 467 (2007)

17. H. Kolsky, Proc. Phys. Soc. B 62, 676 (1949)

18. T. Yokoyama, J. Strain Anal. Eng. Des. 38, 233 (2003)

19. T. Yokoyama, K. Nakai, Int. J. Adhes. Adhes. 56, 13 (2015)

20. B.R.K. Blackman, A.J. Kinloch, F.S. Rodriguez Sanchez, W.S. Teo, J.G. Williams, Eng. Fract. Mech. 76, 2868 (2009)

21. R. Gerlach, C. Kettenbeil, N. Petrinic, Int. J. Impact Eng. 50, 63 (2012)

22. S. Marzi, O. Hesebeck, M. Brede, F. Kleiner, in 7th European LS-DYNA Conference (2009)

23. Livermore Software Technology Corporation, LS-OPT user's manual, version 2.2 (2004)

24. M. Palmares, Strength of hybrid laminates aluminium carbon-fibre joints with different lay-up configurations (University of Porto, Dept. of Mech. Eng., 2016) 